\title{
What is essential is invisible to the eye
}

\author{
Valeria Bisogni, Daniele Piazza, Massimo Puato, Gian Paolo Rossi \\ Department of Medicine-DIMED, I nternal Medicine 4, University Hospital, University of Padua, I taly.
}

Correspondence: Gian Paolo Rossi. Address: Department of Medicine-DIMED, Internal Medicine 4, University Hospital, University of Padua, Via Giustiniani 2, 35126, Padua, Italy. Email: gianpaolo.rossi@unipd.it

Received: January 30, 2014

Accepted: March 11, $2014 \quad$ Online Published: April 8, 2014

DOI : $10.5430 /$ crim.v1n2p75

URL: http://dx.doi.org/10.5430/crim.v1n2p75

\section{Abstract}

A 30 year-old woman was transferred to our Department from the Department of Gynaecology and Obstetrics, where she was admitted for preeclampsia complicated by HELLP syndrome and had undergone therapeutic abortion during the first trimester of pregnancy. She was thereafter diagnosed to have an ACTH-independent Cushing's syndrome, due to an adrenocortical mass.

Hypertension is a common condition during pregnancy and is associated with adverse maternal and perinatal outcomes. It can occur in women with pre-existing chronic hypertension, but can also manifest itself in the second half of pregnancy as new-onset hypertension. The high blood pressure can be due to an underlying cause, but among the possible causes Cushing's syndrome (CS) is very rare during pregnancy, because most of CS patients are infertile. Furthermore, the diagnosis of CS is challenging during pregnancy, because its clinical features may simulate the physiological changes of pregnancy, especially in young women. The case herein reported emphasizes the key role of taking a detailed history and a thorough physical examination for the correct diagnosis, avoiding unnecessary and expensive investigations, and reducing the risk of complications related to a late diagnosis. We herein report also a reappraisal of the prevalence of hypertensive disorders during pregnancy and of the pathophysiology and diagnosis of CS in pregnancy.

\section{Keywords}

Hypertension, Pregnancy, Cushing's syndrome, Adrenal adenoma

\section{I ntroduction}

Cushing's syndrome (CS) in pregnancy was first described in $1953^{[1]}$. Since then only about 140 cases of CS have been reported, which suggests that CS is rare in pregnancy. This could be because hypercortisolism often induces menstrual disturbances that lead to infertility. The most common cause of CS in pregnancy is ACTH-independent adrenal adenoma. For all cases of CS in pregnancy the diagnosis is often challenging because the clinical and biochemical features of CS can mimic those occurring during pregnancy (i.e. high blood pressure, diabetes, rise of 24-h urinary cortisol and morning plasma cortisol, etc.). However, undetected CS during pregnancy carries an excess risk of maternal and foetal complications. Hence, considering the possibility of CS in pregnant women is of utmost importance, particularly in the presence of chronic hypertension, preeclampsia, and other conditions/complications that may endanger the woman and foetal life. 


\section{Case presentation}

\subsection{History}

A 30 years-old young Caucasian woman of was seen on consultation in the Obstetrics and Gynaecology Department, where she was admitted at the 19th week of gestation for preeclampsia complicated by HELLP syndrome. A therapeutic abortion was performed after that she was transferred to our Internal Medicine Department with a diagnosis of "Resistant and complicated arterial hypertension".

On history she reported that high blood pressure (BP) was first detected a month before, at the sixteenth week of gestation, during a medical check-up for uterine bleeding. She had never been taking any drugs therapy before. She reported no history of any significant diseases. A 24 hours ambulatory BP monitoring (ABPM) was performed, in July 2013 that confirmed a mild-to-moderate arterial hypertension with a dipping profile. For this reason she had been prescribed methyldopa (250 mg t.i.d.). A uterine ultrasound showed a detachment of the uterine membranes with hematic effusion. Three weeks later, due to a second episode of uterine bleeding and persistence of high BP, she was re-evaluated and subjected to further investigations. A transthoracic echocardiogram (TTE) showed "marked hypertrophy of the left ventricle" (qualitative report) with "apical obliteration". Labetalol $50 \mathrm{mg}$ /day, nifedipine $30 \mathrm{mg} /$ day and clonidine 300 $\mathrm{mg}$ /day were added to methyldopa. The patient was finally admitted to the Department of Gynaecology and Obstetrics at the 19th week of pregnancy. The investigations performed during hospitalization showed thrombocythemia (PLTS $74 \times$ $10^{3} / \mu \mathrm{L}$ ), signs of hepato-cytolysis (AST $96 \mathrm{U} / \mathrm{L}$, ALT $179 \mathrm{U} / \mathrm{L}$ ) and cholestasis (gGT $214 \mathrm{U} / \mathrm{L}$, alkaline phosphatase 413 $\mathrm{U} / \mathrm{L}$ ), lactate dehydrogenase $417 \mathrm{U} / \mathrm{L}$. The total proteinuria was $470 \mathrm{mg} / 24 \mathrm{~h}$ and eGFR (calculated with MDRD formula) was normal $\left(122 \mathrm{ml} / \mathrm{min} / 1.73 \mathrm{~m}^{2}\right)$. Serum potassium was low $(2.4 \mathrm{mmol} / \mathrm{L})$. Morning serum cortisol was high $(841 \mathrm{nmol} / 1$ n.v. 138 - 690), while serum ACTH was suppressed $(<5 \mathrm{pg} / \mathrm{ml})$. Considering these findings and the persistence of poor BP control, "preeclampsia superimposed on chronic hypertension and HELLP syndrome" was diagnosed. The patient underwent a histerotomy for therapeutic abortion. During the course of all this hospitalization none ever suspected a secondary form of hypertension.

\subsection{Physical examination}

On admission the patient was hemodynamically stable. Her blood pressure was $120 / 70 \mathrm{mmHg}$ while on therapy with potassium canrenoate $50 \mathrm{mg} /$ day, doxazosin $8 \mathrm{mg}$ /day, methyldopa $2 \mathrm{~g} /$ day, nifedipine GITS $60 \mathrm{mg} /$ day, enalapril 20 $\mathrm{mg} /$ day, nebivolol $5 \mathrm{mg} /$ day, isosorbide-5-monohydrate $60 \mathrm{mg} /$ day.

She showed tremor spread, deflected mood, emotional lability, and anxiety. At physical examination she showed most of the physical hallmarks of CS including: a moon face, capillary fragility with widespread petechiae and a superficial hematoma, a thin skin, thin and sparse hair, hirsutism, muscle wasting at arms and legs, a globular abdomen, and striae rubrae in the right quadrant of the abdomen.

\subsection{Biochemical and instrumental investigation}

After the abortion platelet count, indices of hepato-cytolysis, cholestasis, and haemolysis (lactate dehydrogenase), and 24-hour proteinuria normalized, while serum potassium remained low $(2.9 \mathrm{mmol} / \mathrm{L})$. She was then subjected to a series of biochemical and instrumental investigations including plasma renin activity (PRA) and aldosterone supine, baseline and after $50 \mathrm{mg}$ oral captopril challenge, 24-h urinary catecholamines and metanephrines, and thyroid function tests. The search for autoimmune diseases was negative. Since the physical findings and the clinical history of the patient strongly suggested a hypercortisolism we measured $24 \mathrm{~h}$ urinary cortisol excretion that showed very high $(1258 \mathrm{nmol} / 24 \mathrm{~h}$; normal $16-168 \mathrm{nmol} / 24 \mathrm{~h}$ ) values, alongside a basal ACTH level below $5 \mathrm{pg} / \mathrm{ml}$. A 1-mg overnight dexamethasone (Dex) suppression test (Nugent test) showed a morning plasma cortisol of $784 \mathrm{nmol} / \mathrm{L}(<500 \mathrm{nmol} / \mathrm{L})$, which was consistent with a diagnosis of ACTH-independent CS. Abdominal CT imaging showed a left adrenal adenoma, solid, irregular, capsulated and size $87 \mathrm{~mm} \times 70 \mathrm{~mm} \times 70 \mathrm{~mm}$ (see Figure 1). The patient underwent a PET-CT scan that showed net hypermetabolism 
(SUVmax 13, SUVmean 5.5) at the left adrenal mass that presented hypometabolic center (see Figure 2). Laparoscopic left adrenalectomy was performed. Histological examination showed an adrenocortical adenoma mainly composed of zona fasciculata cells of the. BP rapidly normalized after surgery.

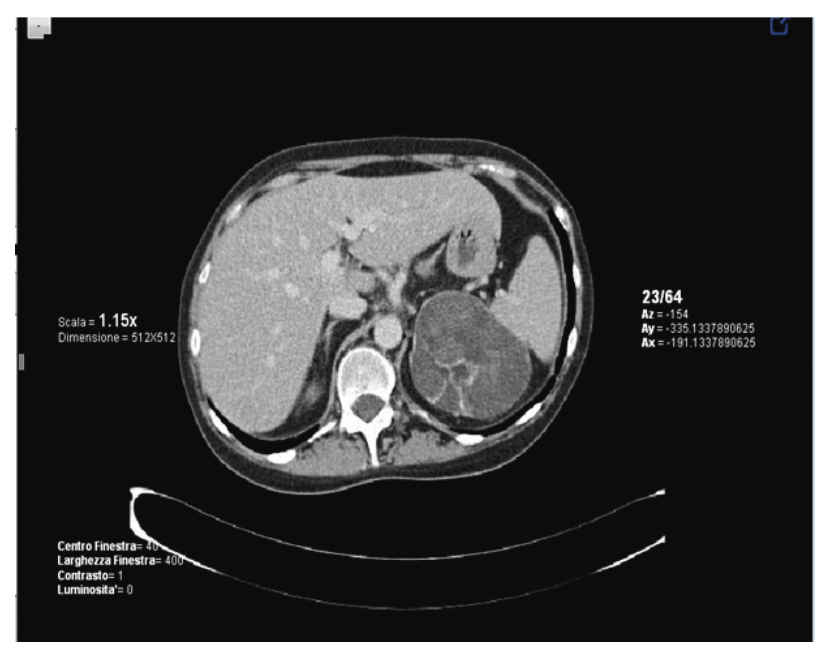

Figure 1. CT images showing a solid mass suggestive of left adrenal adenoma.

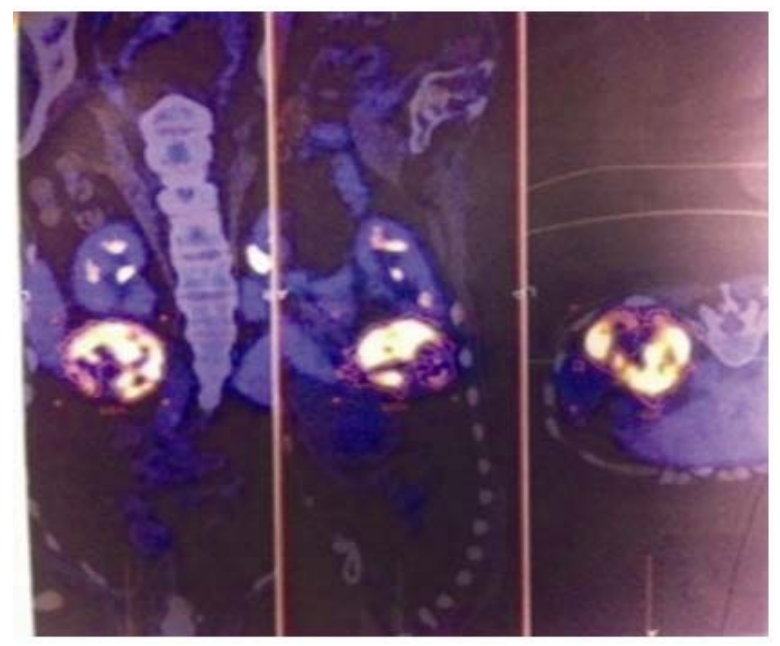

Figure 2. PET-CT scan image: hypermetabolism in the left adrenal gland.

\section{Discussion}

Hypertensive disorders remain a major health issue in pregnancy for both women and their infants. They may occur in women with pre-existing primary or secondary chronic hypertension, and in women who develop new-onset hypertension in the second half of pregnancy. Preeclampsia, either alone or superimposed on pre-existing (chronic) hypertension, is a major risk ${ }^{[1]}$. Hypertensive disorders during pregnancy may occur in women with pre-existing primary or secondary chronic hypertension, and in women who develop new-onset hypertension in the second half of pregnancy. According to the National High Blood Pressure Education Program (NHBPEP) ${ }^{[2]}$ the definition of hypertension during pregnancy is: systolic blood pressure $\geq 140 \mathrm{mmHg}$ and/or diastolic blood pressure $\geq 90 \mathrm{mmHg}$ in most measurements at a distance of several hours in the clinic, Day Hospital or at rest in the Hospital.

The classification of hypertensive disorders is shown in the Table 1. 
Table 1. Classification of hypertensive disorders

\begin{tabular}{|c|c|}
\hline & Definition \\
\hline Preeclampsia (PE) & $\begin{array}{l}\text { Is new hypertension presenting after } 20 \text { weeks of pregnancy with } \\
\text { significant proteinuria ( } \geq 300 \mathrm{mg} / 24 \mathrm{~h} \text { ) in a woman previously } \\
\text { normotensive. }\end{array}$ \\
\hline Chronic hypertension & $\begin{array}{l}\text { Is hypertension that is present at the booking visit or before } 20 \\
\text { weeks or if the woman is already taking antihypertensive } \\
\text { medication when referred to maternity services. It can be primary or } \\
\text { secondary in aetiology. }\end{array}$ \\
\hline Preeclampsia superimposed on chronic hypertension & $\begin{array}{l}\text { Is the appearance of de novo proteinuria starting with gestational } \\
\text { week } 20 \text {, more or less associated with: a sudden increase of blood } \\
\text { pressure, the appearance of thrombocytopenia, abnormal levels of } \\
\text { transaminases, renal failure, sign and/or symptoms of neurological } \\
\text { disorders (i.e. seizures, headache, persistent scotoma, eclampsia), } \\
\text { slowdown of fetal growth. }\end{array}$ \\
\hline Gestational hypertension & $\begin{array}{l}\text { Is new hypertension presenting after } 20 \text { weeks without significant } \\
\text { proteinuria; generally the pressure normalizes postpartum. }\end{array}$ \\
\hline
\end{tabular}

The guidelines also includes the definition of HELLP (Hemolysis Elevated Liver enzyme levels Low Platelet count) syndrome as a condition characterized by haemolysis, elevated liver enzymes, and low platelet count. HELLP occurs early in the first trimester in $0.2 \%-0.8 \%$ of pregnancies and in $70 \%-80 \%$ coexists with $\mathrm{PE}^{[3]}$. Main diagnostic criteria are, according to Tennessee Classification:

[1] Platelets $\leq 100.000 \times 10^{3} / \mu \mathrm{L}$;

[2] $\quad \mathrm{AST} \geq 70 \mathrm{U} / \mathrm{L}$;

[3] $\quad \mathrm{LDH} \geq 600 \mathrm{U} / \mathrm{L}$.

The syndrome is a progressive condition with frequent and serious complications ${ }^{[4]}$. The hypertension can be most commonly essential, or secondary to a recognizable cause. Amongst the latter CS is exceedingly rare. (about $0.1 \%$ of all forms), likely because hypercortisolism prevents normal follicular development and ovulation, and is also associated with significant morbidity and mortality, compared to pregnancy without complication. The most common complications of CS in pregnancy are hypertension and impaired glucose tolerance or diabetes mellitus. More rarely CS in pregnancy is associated with poor wound healing, preeclampsia, osteoporosis, severe psychiatric complications, maternal cardiac failure, and death. Adrenocortical adenomas represent about $40 \%-50 \%$ of CS cases in pregnancy, a rate much higher than the $15 \%$ found in non-pregnant women. On the other hand, Cushing's disease (CD), caused by a pituitary adenoma comprises only over $30 \%$ of cases in pregnant women, as compared with $58 \%-70 \%$ in non-pregnant women (see Table 2). Of the remaining cases, about $10 \%$ are due to adrenal carcinoma, with the rest due to ACTH-independent hyperplasia, ectopic ACTH secretion, and unspecified causes.

Table 2. Etiology of CS in pregnancy vs non-in pregnancy

\begin{tabular}{lcc}
\hline \multicolumn{1}{c}{ Etiology } & Pregnant women (\%) & Non-pregnant women (\%) \\
\hline CD (pituitary adenoma) & 33 & 61 \\
Adrenal adenoma & 46 & 15 \\
Carcinoma & 10 & 8 \\
ACTH-independent hyperplasia & 3 & 2 \\
Ectopic CS & 3 & 12 \\
Unspecified & 5 & 2 \\
\hline
\end{tabular}


The difficulty of diagnosis of hypercortisolism during pregnancy can be intriguing as the clinical features. In pregnant women with CS (central weight gain, oedema, fatigue, emotional upset, hypertension and glucose intolerance, bruising, and hirustism) are also common to pregnancy. Moreover, pregnancy has a profound effect on adrenal steroidogenesis, even though the maternal adrenal glands do not change morphologically during pregnancy. At variance with the hypothalamic-pituitary-adrenal (HPA) axis, glucocorticoid levels exert a positive feedback on the placental corticosteroid axis. Corticotropin Releasing Hormone (CRH), which modulates both maternal and fetal pituitary-adrenal axes, rises several hundred-fold during pregnancy. Both maternal and placental ACTH and cortisol levels rise markedly during pregnancy with the initial surge at the 11th week of gestation, a further significant rise after 16-20 weeks gestation, and a final surge during labor. In addition, the feto-placental unit has a marked capacity for steroidogenesis, causing plasma cortisol levels to rise 2-3 fold over the course of the pregnancy above the levels of non-pregnant controls, reaching values that are typically in the range seen in CS. Twenty-four hours urinary cortisol excretion parallels the rise in serum cortisol.

Furthermore, placenta-derived oestrogens stimulate the hepatic production of Cortisol Binding Globulin (CBG) levels, thus increasing total cortisol levels and decreasing cortisol clearance. Despite the increase in placental hormones and increased HPA axis function, a normal maternal circadian rhythm of ACTH persists throughout pregnancy ${ }^{[1]}$. The first line tests for diagnosing CS according to the Endocrine Society Clinical Practice Guidelines ${ }^{[5]}$, are measurement of 24-h urinary cortisol and the 1-mg overnight dexamethasone (Dex) suppression test. As discussed earlier, the usefulness of the 1-mg overnight dexamethasone suppression test is more limited in pregnant women than in the general population. The guidelines also warn against the use of ACTH levels and the tests for the subtyping of CS. Radiographic studies, such as CT scan, which are important when there is biochemical evidence of CS are unfeasible in pregnancy, MR being the only option for imaging the disease. Medical treatment with metyrapone, ketoconazole, cyproheptadine may be considered in patients with contraindications to surgical therapy.

Most cases of CS in pregnancy described thus far mainly occurred during the early third trimester of pregnancy. As mentioned above, the complications of CS in pregnancy are many, as testified by the present case that developed preeclampsia and HELLP syndrome and also echocardiographic signs of LVH. These complications often lead to premature interruption of pregnancy.

We would like here to underline the crucial role of a careful physical examination. In the present case an early recognition of the features of CS could have led to an earlier diagnosis and appropriate management, possibly avoiding miscarriage.

\section{Acknowledgements}

Dr Bisogni, Piazza and Puato managed the case under the supervision of Professor Rossi, and they participated also in the acquisition of the data. Dr Bisogni drafted the manuscript and Dr Piazza participated in drafting the article. Professor Rossi revised it.

\section{References}

[1] Lindsay JR and Nieman LK. The Hypothalamic-Pituitary-Adrenal Axis in Pregnancy: Challenges in Disease Detection and Treatment. Endocrine Reviews. 26: 775-799. http://dx.doi.org/10.1210/er.2004-0025

[2] Report of the National High Blood Pressure Education Program Working Group on High Blood Pressure in Pregnancy. Am. J. Obstet. Gynecol. 2000; 183, S1-22.

[3] Abildgaard U, Heimdal K. Pathogenesis of the syndrome of hemolysis, elevated liver enzymes, and low platelet count (HELLP): a review. Eur J Obstet Gynecol Reprod Biol. 2013 Feb; 166: 117-23. http://dx.doi.org/10.1016/j.ejogrb.2012.09.026

[4] Haram K, Svendsen E, Abildgaard U. The HELLP syndrome: clinical issues and management. A Review. BMC Pregnancy Childbirth. 2009; 9: 8. http://dx.doi.org/10.1186/1471-2393-9-8

[5] Guignat L, Bertherat J. The diagnosis of Cushing's syndrome: an Endocrine Society Clinical Practice Guideline: commentary from a European perspective. European Journal of Endocrinology. 2010; 163: 9-13. http://dx.doi.org/10.1530/EJE-09-0627

Published by Sciedu Press 